\title{
Progress with Closed-loop Systems in Type 1 Diabetes
}

\author{
Lalantha Leelarathna, MBBS, MRCP(UK), MSC ${ }^{1}$ and Roman Hovorka, BSc, MSc, PhD ${ }^{2}$ \\ 1. Clinical Research Associate; 2. Principal Research Associate, \\ Institute of Metabolic Science, University of Cambridge
}

DOI: $10.17925 /$ USE.2010.06.1.58

\begin{abstract}
Automated insulin delivery by means of a glucose-responsive closed-loop system has often been cited as the 'holy grail' of type 1 diabetes management. Reflecting the technological advances in interstitial glucose measurements and wider use of continuous glucose monitoring, recent research in closed-loop glucose control has focused on the subcutaneous route for glucose measurements and insulin delivery. The primary aim of such systems is to keep blood glucose within the target range while minimizing the risk of hypoglycemia with minimal input from the user. This article examines recent developments in the field of interstitial glucose measurement, limitations of the current generation of devices and implications on the performance of closed-loop systems. Clinical results and the advantages and disadvantages of different closed-loop configurations are summarized. Potential future advances in closed-loop systems are highlighted.
\end{abstract}

\section{Keywords}

Closed-loop system, artificial pancreas, continuous glucose monitoring, type 1 diabetes, hypoglycemia

Disclosure: The authors have no conflicts of interest to declare.

Acknowledgements: The authors acknowledge support from the Juvenile Diabetes Research Foundation (\#22-2006-1113, \#22-2007-1801, \#22-2009-801), Diabetes UK (BDA07/0003549, BDA07/0003551), EC FP7 (247138), National Institutes for Health (NIH) (DK085621), and National Institute for Health Research (NIHR) Cambridge Biomedical Research Centre.

Received: August 2, 2010 Accepted: September 20, 2010 Citation: US Endocrinology, 2010;6:58-62

Correspondence: Roman Hovorka, BSC, MSC, PhD, Institute of Metabolic Science, University of Cambridge, Box 289, Addenbrooke's Hospital, Hills Road, Cambridge CB2 0QQ,

UK. E: rh347@cam.ac.uk

Type 1 diabetes is one of the most common endocrine problems in childhood and adolescence and its incidence is increasing. ${ }^{1,2}$ of the estimated 480,000 children with type 1 diabetes worldwide, nearly a quarter come from the European region, with a steeper increase in incidence in some of the Central and Eastern European countries. ${ }^{3}$ While type 1 diabetes only accounts for $5-10 \%{ }^{4}$ of the 285 million people with diabetes in 2010, it remains a serious chronic disorder with increased morbidity, mortality and reduced quality of life. ${ }^{5}$

The risk for long-term complications, both microvascular and cardiovascular could be reduced by improving glycemic control. ${ }^{6,7}$ Despite the availability of therapeutic options such as self-monitoring of blood glucose, structured patient education, rapid-acting insulin analogs and insulin pump therapy, glycemic control in the majority of patients with type 1 diabetes remains suboptimal. The biggest barrier to intensification of control is the increased risk for hypoglycemia. ${ }^{8}$ Nocturnal hypoglycemia was common (occurring on $8.5 \%$ of nights) and often prolonged (duration $\geq 2$ hours on $23 \%$ of nights) during the recently concluded Juvenile Diabetes Research Foundation (JDRF) continuous glucose monitoring (CGM) study. ${ }^{9}$ Even in patients with good control, as judged by average glycated haemoglobin $\left(\mathrm{HbA}_{1 \mathrm{c}}\right)$, significant glucose excursions occur with periods of silent hyper- and hypoglycemia. ${ }^{10,11}$ The development of an automated closed-loop system—also referred to as an artificial electromechanical endocrine pancreas-that could overcome the limitations of modern diabetes therapy has long been an aspiration of the diabetes community. The objective of this article is to describe the current state-of-the-art, challenges, and future directions for closed-loop systems.

\section{Components of Closed-Ioop Insulin Delivery Systems}

The generic definition of 'closed-loop' is that it is an automatic control system in which an operation, process, or mechanism is regulated by feedback. In type 1 diabetes, closed-loop systems continually modulate insulin delivery according to prevailing glucose levels. This is in contrast with currently available conventional insulin pump therapy, whereby insulin is delivered at pre-programmed rates and only intermittently adjusted. Closed-loop systems comprise three main components (see Figure 1): a CGM device, a control algorithm that determines the insulin delivery rate, and a portable electromechanical insulin pump.

For practical reasons, the subcutaneous approach for continuous glucose sensing and insulin delivery has become the preferred mode of operation. Other modes, such as the intravenous approach for glucose sensing and insulin delivery, may be of use for example in intensive care settings. 


\section{Continuous Glucose Monitoring}

CGM devices consist of a disposable sensor that is implanted into the subcutaneous tissue, a non-implanted transmitter that powers the sensor and transfers data wirelessly, and a hand-held receiver. The sensors used in the commercially-available devices contain the enzyme glucose oxidase, which converts glucose into gluconic acid and hydrogen peroxide. ${ }^{12}$ The resulting changes in electrical charge are proportional to the concentration of glucose. The signal is transmitted to the hand-held receiver, which converts the measured current into glucose values using a transformation algorithm using one or more reference glucose values obtained via finger-prick glucose testing. This process is commonly referred to as calibration. The receiver also acts as the user interface, with information on glucose data updated every one to 10 minutes, a graphical display of glucose levels for user-specified time periods and also glucose trend arrows and alarms alerting the user to impending hyper- or hypoglycemia. Information on hyper- and hypoglycemia is particularly useful as the direction of glucose drift will allow the user to take precautionary action before a significant event occurs. Receivers can be either standalone devices (such as Guardian RT, Freestyle Navigator and Dexcom) or incorporated into insulin pumps (Medtronic Veo pump with MiniLink Sensor).

The landmark JDRF-funded CGM study ${ }^{13}$ showed that in adults $\geq 25$ years of age, CGM significantly reduced the mean $\mathrm{HbA}_{1 \mathrm{c}}$ level compared with home monitoring at 26 weeks (mean difference in change, $-0.53 \%$, 95\% confidence interval $[\mathrm{Cl}]-0.71$ to $-0.35 ; \mathrm{p}<0.001)$. It also found that CGM significantly increased the proportion of people who achieved a target $\mathrm{HbA}_{1 \mathrm{c}}$ level of $<7.0 \%$ and significantly increased the time spent within the target glucose range of $3.9-10 \mathrm{mmol} / \mathrm{l}$. No such benefit was seen in the 15-24 or 8-14 years of age groups. ${ }^{13}$ Compared with adults, CGM was significantly less frequently used in these age groups. Factors such as the limited ability or willingness of patients to use the information provided by the CGM remains a possible reason. ${ }^{14}$ In further investigations in patients with tight glycemic control $\left(\mathrm{HbA}_{1 \mathrm{c}}<7.0\right)$, the use of CGM reduced the time spent in hypoglycemia without worsening glucose control. ${ }^{15}$ Patients aged 25 years or more received further follow-up and these beneficial effects were observed at 12 months. ${ }^{16}$

Recent studies have shown the utility of combining sensors with insulin pumps: so-called sensor-augmented pump therapy. The sensor-augmented group achieved better control over those who used multiple daily injections. The baseline mean $\mathrm{HbA}_{1 \mathrm{c}}$ level was $8.3 \%$ in the two study groups. It decreased to $7.5 \%$ in the sensor-augmented pump-therapy group compared with $8.1 \%$ in the multiple daily injection group at 12 months $(p<0.001){ }^{17}$ Further randomized studies that compare sensor-augmented pump therapy with standard insulin-pump therapy are required to unravel the impact of glucose sensing during continuous subcutaneous insulin infusion.

Despite significant advances in CGM technology, reliability and accuracy remain a concern and are often regarded as the rate-limiting step in progression to fully automated closed-loop insulin delivery. ${ }^{18,19}$ A time lag exists between glucose values obtained by CGM and reference glucose levels. The time lag comprises the physiological delay between changes in blood glucose and interstitial fluid glucose levels. Further delay in

\section{Figure 1: The Components of a Closed-loop Insulin Delivery System}

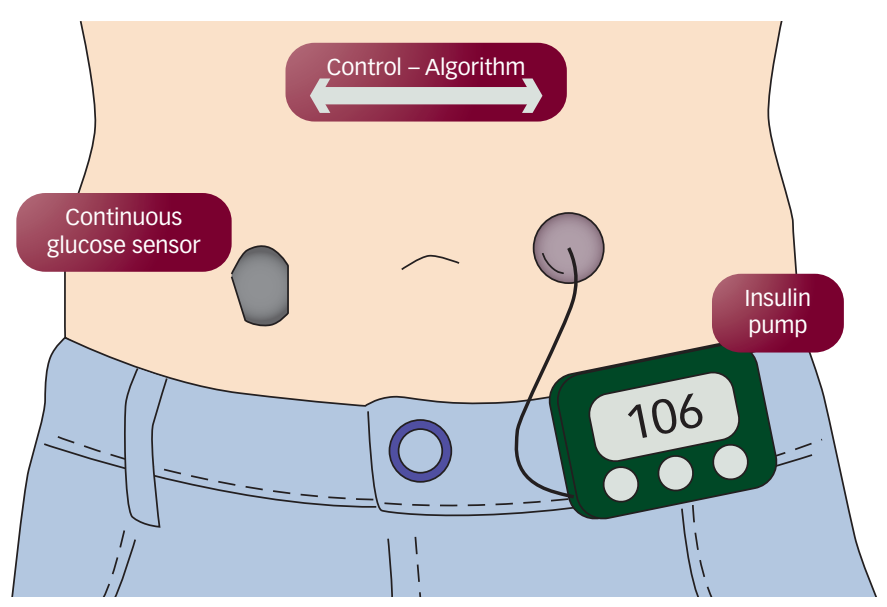

Courtesy of Juvenile Diabetes Research Foundation (JDRF).

sensor glucose occurs due to data filtering involved in removing noise from the measured electric current. The reported time lag for the three commonly used CGM devices is between five and seven minutes (Dexcom Seven Plus) ${ }^{20,21}$ and eight to 15 minutes for the Freestyle Navigator ${ }^{22,23}$ and Guardian RT devices. ${ }^{24,25}$ Studies performed using modern CGM devices have shown the overall accuracy measured by mean absolute relative difference to be around $15 \% .{ }^{26-28}$ In addition to the time lag, sensors are prone to transient and persistent deviations from the reference glucose and, at times, a complete loss of data. The reported frequency of such unreliable data points have varied from seven to $30 \% .{ }^{28}$ The most common reasons for transient glucose deviations of one to four hours are either due to temporal loss/increase in sensitivity of the sensor or mechanical perturbations including sensor dislodgement. ${ }^{29}$ When a sensor under-reads blood glucose, this artefact is called 'drop-out' and poses little risk. Temporal insulin underdelivery may occur due to drop-out. However, the opposite situation, where the sensor over-reads blood glucose, will lead to higher insulin rates with the potential risk for hypoglycemia. Persistent sensor glucose deviations are also caused by erroneous calibration finger-stick glucose measurements and pose the greatest challenge to safe closed-loop operation. Persistent deviations occur between two consecutive calibration points and may last for 12-48 hours. Up to 100\% calibration error has been recorded. ${ }^{30}$ Despite these limitations, a reported risk analysis suggests that closed-loop systems may substantially reduce, but not eliminate, the risk for severe nocturnal hypoglycemia. ${ }^{30}$

\section{Control Algorithms}

The role of the control algorithm is to translate, in realtime, the information it receives from the glucose monitor and to compute the amount of insulin to be delivered by the insulin pump. Only a brief description of control algorithms is provided here. A more detailed account can be found elsewhere. ${ }^{31}$ There are two main categories of control algorithms, i.e the model predictive control (MPC) ${ }^{32-34}$ and classic feedback control embodied in the proportional-integral derivative (PID) controller. ${ }^{35-38} \mathrm{MPC}$ is at the forefront of current research with contributions, for example, by Wang et al., ${ }^{39}$ El-Khatib et al., ${ }^{40}$ Lee et al., ${ }^{41}$ and Hovorka et al. ${ }^{32}$ The vital component of MPC is a model linking insulin 
Figure 2: Plasma Glucose During Closed-loop and Conventional Therapy

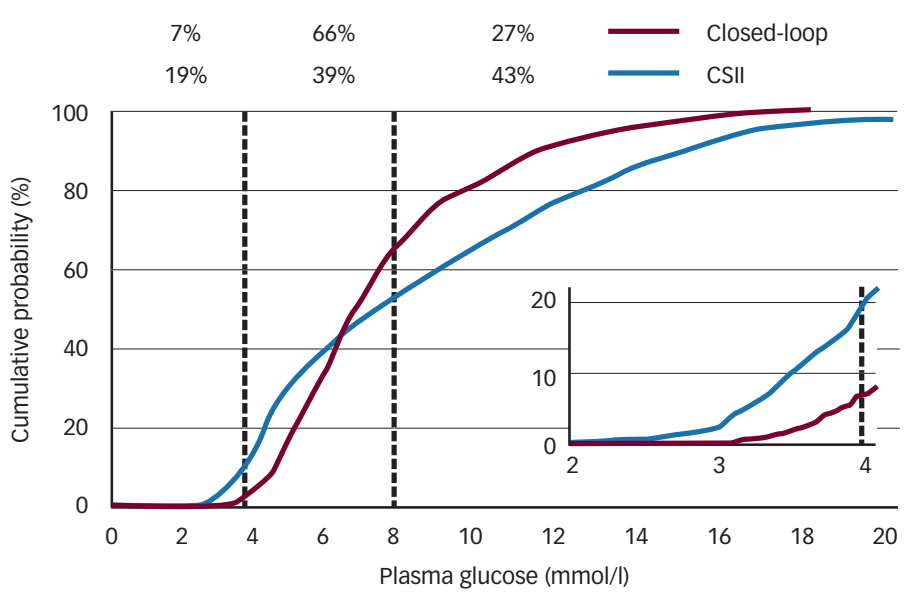

Cumulative probability of plasma glucose concentrations during closed-loop (CL) insulin delivery and continuous subcutaneous insulin infusion (CSII) obtained during overnight studies in Cambridge in children and adolescents. The vertical dashed lines denote the target glucose range (3.91-8.00mmol/I). The inset shows detail at low plasma glucose concentrations. The numbers in rows are the total percentage time for which plasma glucose was lower than, within, or higher than the target range from the start of $C L$ control at 8 pm until 8 am the next day. Reproduced with permission. ${ }^{42}$

infusion and meal ingestion to glucose excursions. The MPC approach can handle delays associated with insulin absorption and take into account meal intake and prandial boluses delivered manually by the patient. By contrast, the PID controller adjusts the insulin infusion rate by assessing glucose excursions from three viewpoints: the departure from the target glucose level (the proportional component); the rate of change in measured glucose (the derivative component); and the area-under-curve between measured and target glucose (the integral component). Control algorithms can be initialized by clinically relevant information, such as subject's weight, ${ }^{40}$ total daily insulin dose and preprogrammed basal rate. ${ }^{42}$ These algorithms can adapt themselves to time-varying insulin needs in realtime based on sensor glucose levels. ${ }^{40}$ However, most algorithms adopt a non-adaptive approach using offline initialization. Further research is needed to determine the optimum frequency of adaptation.

\section{Clinical Studies of Closed-loop Insulin Delivery Systems} Suspended Insulin Delivery

The simplest form of a closed-loop system is to suspend insulin delivery when the patient reaches a hypoglycemic state. Introduced in 2009, the Medtronic Veo pump coupled with a CGM sensor suspends insulin delivery for up to two hours if hypoglycemia alarms are not acknowledged. The main objective of this approach is to reduce the severity and duration, but not the incidence, of hypoglycemia. Recent work by Dassau et al. ${ }^{43}$ and Buckingham et al. ${ }^{44}$ highlights the development and use of a more advanced approach combining five algorithms to predict and prevent hypoglycemia. During the latter study, ${ }^{44}$ in the first 14 subjects hypoglycemia was induced by gradually increasing the basal insulin infusion rate without the use of pump shut-off algorithms. During the subsequent 26 patient studies, insulin suspension was initiated at normoglycemia when sensor glucose was decreasing and two or three algorithms predicted hypoglycemia. A 35-minute prediction horizon was used with a glucose threshold of $3.9 \mathrm{mmol} / \mathrm{l}$ to predict impending hypoglycemia. The pump shut-off lasted for up to two hours. This approach prevented hypoglycemia $(<3.3 \mathrm{mmol} / \mathrm{l})$ on $75 \%$ of nights (and reduced events by $84 \%$ ) without hyperglycemia rebound. One of the major concerns about the automated suspension of insulin delivery is the subsequent risk for hyperglycemia. The Institute of Metabolic Science group and others have demonstrated that such pump suspensions are safe as part of a closed-loop operation in children and adolescents. ${ }^{45,46}$ Elleri et al. ${ }^{46}$ showed that during insulin delivery suspension lasting $\geq 90$ minutes, normal glucose levels were maintained with physiological levels of plasma insulin throughout, with a nadir plasma insulin level of $119 \pm 78 \mathrm{pmol} / \mathrm{l}$. Plasma glucose was $6.2 \pm 3.2 \mathrm{mmol} / \mathrm{l}$ at the time of interruption and $5.5 \pm 2.0 \mathrm{mmol} / \mathrm{I} 105$ minutes later. Plasma glucose declined during the first hour of the interruption at a rate of $0.02 \pm 0.03 \mathrm{mmol} / / /$ minute and reached a nadir of $5.2 \pm 2.7 \mathrm{mmol} / \mathrm{l}$. Plasma glucose started to increase at a rate of $0.01 \pm 0.03 \mathrm{mmol} / \mathrm{I} / \mathrm{minute}$ 105 minutes after the interruption. When insulin delivery restarted, plasma glucose was $6.4 \pm 2.2 \mathrm{mmol} / /$ and peaked at $7.9 \pm 2.1 \mathrm{mmol} / \mathrm{l}$ in 60 minutes.

\section{Overnight Closed-loop Control}

Sleep is a recognized risk factor for severe hypoglycemia due to absent warning symptoms and blunted sympatho-adrenal response. ${ }^{47}$ Nocturnal hypoglycemia is therefore very common but often asymptomatic and undetected-an observation that has been confirmed by the use of CGM. ${ }^{9}$ Furthermore, $75 \%$ of hypoglycemic seizures in children occur during sleep. ${ }^{48}$ Severe nocturnal hypoglycemia may be implicated in dead-in-bed syndrome. ${ }^{49,50}$ since overnight glucose control is not challenged by exercise or meals, overnight closed-loop control is a simpler but important step towards the goal of an artificial pancreas.

\section{Cross-over Studies}

Randomized cross-over studies evaluating overnight closed-loop control in children, adults and pregnant women with type 1 diabetes have been performed at the Institute of Metabolic Science. Results in children and adolescents have been published recently. ${ }^{42}$ During 33 closed-loop nights, every 15 minutes sensor glucose values were fed into an MPC controller, which calculated the insulin-infusion rate. The insulin pump was adjusted manually by a research nurse. During 22 control nights, the standard insulin pump settings of subjects were applied. An analysis of pooled data documented increased time in the target glucose range between 3.9-8.0mmol/I (60 versus 40\%) and reduced time that glucose levels were $<3.9 \mathrm{mmol} / \mathrm{l}$ (2.1 versus $4.1 \%$ ) (see Figure 2). ${ }^{42}$ The closed-loop system reduced the frequency of plasma glucose levels $<3.3 \mathrm{mmol} / \mathrm{I}$ from 7.5 to $0.7 \%$. No events with plasma glucose concentration $<3.0 \mathrm{mmol} / \mathrm{I}$ were recorded during closed-loop delivery compared with nine events during standard treatment. No rescue carbohydrates were given during closed-loop nights. Average overnight insulin delivery was similar during closed-loop and standard treatment. ${ }^{42}$ The preliminary results in adults ${ }^{51,52}$ and pregnant women ${ }^{53}$ are also promising.

\section{Day and Night Closed-loop Control}

Providing closed-loop control during the daytime is more challenging due to the glucose excursions resulting from varying diet and exercise patterns. Delays involved in the absorption of rapid-acting insulin analogs pose further limitations in the effective implementation of 
closed-loop control during the post-prandial period. For example, in early closed-loop feasibility studies, post-prandial hyperglycemia resulted in insulin overdelivery leading to late post-prandial hypoglycemia requiring rescue carbohydrates. ${ }^{54}$ One of the ways to minimize post-prandial hypoglycemia is to announce the meal. In a subsequent study conducted by the Yale group, the fully closed-loop approach without meal announcement was tested against the meal announcement approach accompanied with a small prandial insulin bolus 10-15 minutes before the meal..$^{55}$ This study was performed using Medtronics ePID system using a proportional integral derivative controller in 17 well-controlled adolescents over 34 hours of closed-loop control. The meal announcement approach tended to improve post-prandial glucose levels (peak $10.8 \pm 2.6$ versus $12.5 \pm 2.8 \mathrm{mmol} / \mathrm{l}$ ) and mean glucose levels $(7.5 \pm 2.5$ versus $7.8 \pm 3.1 \mathrm{mmol} / \mathrm{l}){ }^{55}$ The overall night glucose levels and associated standard deviations were excellent $(6.2 \pm 1.5 \mathrm{mmol} / \mathrm{l})$. In the last 24 hours of closed-loop control, three nocturnal hypoglycemic events $(<3.3 \mathrm{mmol} / \mathrm{l})$ were observed. ${ }^{55}$ In a further study using a model-predictive controller developed by Magni et al., day and night closed-loop control was tested in 14 adults with well-controlled type 1 diabetes. ${ }^{56,57}$ Closed-loop control reduced the frequency of nocturnal hypoglycemia events compared with conventional treatment $(<3.9 \mathrm{mmol} / \mathrm{l}$, five versus 17 events). There was also a tendency of increasing time spent in the target glucose range $(3.9-7.8 \mathrm{mmol} / \mathrm{l})$. After breakfast, the closed-loop system controlled glucose levels as effectively as patient-directed conventional insulin pump therapy.

Studies presented so far have been performed using either PID or MPC controllers. In a recent pilot study by Phillip et al., a novel fuzzy logic controller was used in a fully closed-loop fashion in seven patients with well-controlled type 1 diabetes. ${ }^{58}$ Data collected over three to five days were used to individualize the fuzzy logic algorithm. During a 24-hour period with three standardized meals containing between $17.5 \mathrm{~g}$ and $70 \mathrm{~g}$ of carbohydrates, $73 \%$ of the sensor values ranged between $3.9-10 \mathrm{mmol} / \mathrm{l}, 27 \%$ were $>10 \mathrm{mmol} / \mathrm{l}$, and none were $<3.9 \mathrm{mmol} / \mathrm{l}^{58}$ There were no symptomatic hypoglycemic events. Further studies are planned under daily life conditions.

\section{Dual Hormone Closed-loop}

It may be possible to improve the performance of closed-loop systems by the use of other hormones in addition to insulin. Damiano et al. ${ }^{59}$ and Ward et al. ${ }^{60}$ have investigated the use of glucagon co-administration to reduce the risk for hypoglycemia. In the former study by Damiano et al., an MPC controller was used for insulin and a PID controller for glucagon administration. No hypoglycemia $(<3.9 \mathrm{mmol} / \mathrm{l})$ was observed during 24 hours of fully closed-loop in 11 adults once an appropriate model of insulin absorption was used.59 Insulin and glucagon delivery were dependent on blood glucose and further investigation evaluating the approach with sensor glucose values are warranted. In the study by Ward et al. ${ }^{60}$ use of glucagon resulted in significantly less time spent in the hypoglycemic range ( $15 \pm 6$ versus $40 \pm 10$ minutes/day). In addition, use of glucagon in high-gain pulses were more effective than slow, prolonged infusion. This reduced the frequency of hypoglycemic events $(1.0 \pm 0.6$ versus $2.1 \pm 0.6$ events/day) and the need for carbohydrate treatment $\left(1.4 \pm 0.8\right.$ versus $4.0 \pm 1.4$ treatments/day). ${ }^{60}$ Further studies are under way to examine the effect of other hormones, such as pramlintide. The limitations of using a multihormone approach include the need for dual-chamber insulin pumps, the need for stable glucagon analogs, and the need for two delivery catheters.

\section{Intraperitoneal Insulin Delivery}

Reported benefits of intraperitoneal insulin delivery include fast insulin action and reduced variability of insulin absorption. Renard et al. ${ }^{61,62}$ have examined the feasibility and efficacy of intraperitoneal insulin delivery over two days in eight adults with an implanted insulin pump driven by subcutaneous glucose sensor using a PID algorithm (ePID Medtronic system) with pre-meal insulin dosing. Excluding two early post-prandial hours, the closed-loop system achieved a higher percentage of time in the $4.4-6.6 \mathrm{mmol} / /$ range $(46 \pm 5$ versus $29 \pm 7 \%$ ) and lower mean blood glucose levels $(6.9 \pm 0.8$ versus $7.9 \pm 1.6 \mathrm{mmol} / \mathrm{l}) .{ }^{61}$ Time spent with blood glucose $<3.3 \mathrm{mmol} / \mathrm{l}$ was low and similar for both closed-loop and conventional treatment.

\section{Simulators}

The development, evaluation, and testing of closed-loop systems is time-consuming and costly. Testing in a computer-based environment with a collection of virtual subjects may provide valuable information. This idea was originally advocated by chassin et al..$^{63}$ The Institute of Metabolic Science ${ }^{64}$ and other groups ${ }^{65}$ have developed simulators that could be used in variety of aspects, such as evaluation of control algorithms, ${ }^{33}$ sensor errors and sensor time $\operatorname{lag}_{1}{ }^{30}$ errors in pump delivery, ${ }^{66}$ size of the control step, ${ }^{67}$ pump occlusion, ${ }^{63}$ exercise, ${ }^{68}$ and unannounced meals. Data obtained from such studies could also be used to expedite approval from regulatory bodies. ${ }^{65}$

\section{Challenges and the Way Forward}

Despite the progress that has been made in closed-loop insulin delivery over the last decade, challenges remain. Accuracy and reliability of CGM is often considered a bottleneck and further improvements may facilitate the increased safety and efficacy of closed-loop performance. With current rapid-acting insulin analogs, a substantial delay exists between subcutaneous insulin delivery and the reduction in blood glucose. It may take 90-120 minutes to reach the maximum extent of blood glucose-lowering after administration of a subcutaneous bolus of a rapid-acting insulin analog. ${ }^{18}$ This is often underappreciated.

In a fully closed-loop system without meal announcement, the controller depends on the rate of glucose increase for the delivery of an insulin bolus, with a risk for overaggressive insulin delivery during post-prandial peaks. In order to prevent hypoglycemia, high glucose levels have to be normalized slowly, even during closed-loop periods. The means to account for insulin on-board during closed-loop control have been suggested as a safety feature. ${ }^{69}$ So far, studies have used the slightly less ambitious semi-automated approach of meal announcement and use of a pre-meal bolus. Ultrafast-acting insulin analogs or other means to accelerate insulin absorption or, alternatively, reversibly and systematically slow down the absorption of subcutaneously administered insulin would provide the greatest additional benefit. They can facilitate greater physiological prandial glucose control and may allow safe and efficacious fully closed-loop control. Human hyaluronidase co-administration with insulin is promising. ${ }^{70,71}$ Similarly, VIAject insulin appears to be absorbed faster than existing rapid-acting analogs. ${ }^{72}$ 
Local heating has been proposed to accelerate insulin absorption ${ }^{73}$ as well as dermal insulin delivery. The pharmacokinetic and pharmacodynamic characteristics of rapid-acting insulin analogues vary between and within subjects. ${ }^{74} \mathrm{An}$ incorrect assumption about insulin pharmacokinetics may lead to insulin overdosing and late post-prandial hypoglycemia during closed-loop control. ${ }^{59}$ Up to four-fold between-subject variability in lispro pharmacokinetics has been observed with occasionally as much as a $50 \%$ within-subject variability on repeated occasions. ${ }^{60}$ A more modest 20-25\% within-subject variability has been reported in healthy subjects under controlled conditions. ${ }^{74}$

A further challenge to closed-loop systems is the within-subject variability of insulin needs. These include day-to-day but also to hour-to-hour variations in insulin sensitivity due to circadian and diurnal cycles, dawn phenomenon, acute illness, stress, exercise, and the delayed effect of alcohol. Despite these limitations, the Institute of Metabolic Science group and others have demonstrated that closed-loop systems under controlled conditions are superior to standard insulin-pump therapy. The former improve the time spent in the target glucose range and reduce time spent below the target level. The next challenge is to demonstrate the feasibility, safety, and efficacy of such systems under free-living conditions. The Institute of Metabolic Science group believes that safe and efficacious overnight closed-loop control under free-living conditions can be achieved with currently available 'off-the-shelf' CGM devices and insulin pumps.

\section{Conclusions}

The development of a safe and reliable automated closed-loop insulin delivery system has long been considered the holy grail of type 1 diabetes management. Aided by developments in CGM technology and coupled with advanced algorithms, such closed-loop insulin delivery systems have made significant progress over the last few years, with small-scale studies showing superior performance in comparison with current best-available therapy. The introduction of such systems into clinical practice will be a phased process, with early models focussing on reduction of the burden of hypoglycemia, especially in vulnerable groups, such as children. There is a need for larger studies under free-living conditions before such systems can be approved for general use. Day and night fully closed-loop control is likely to be more challenging. Further refinements may be required in CGM reliability and performance, as well as the development of more rapidly-acting insulins. There is also a need to improve the convenience for the user by miniaturizing the devices. Work is under way to develop a single-port device where glucose sensing and insulin delivery could be undertaken simultaneously. The success of this project will require greater collaboration between the medical device industry, regulatory authorities, and the wider scientific community.

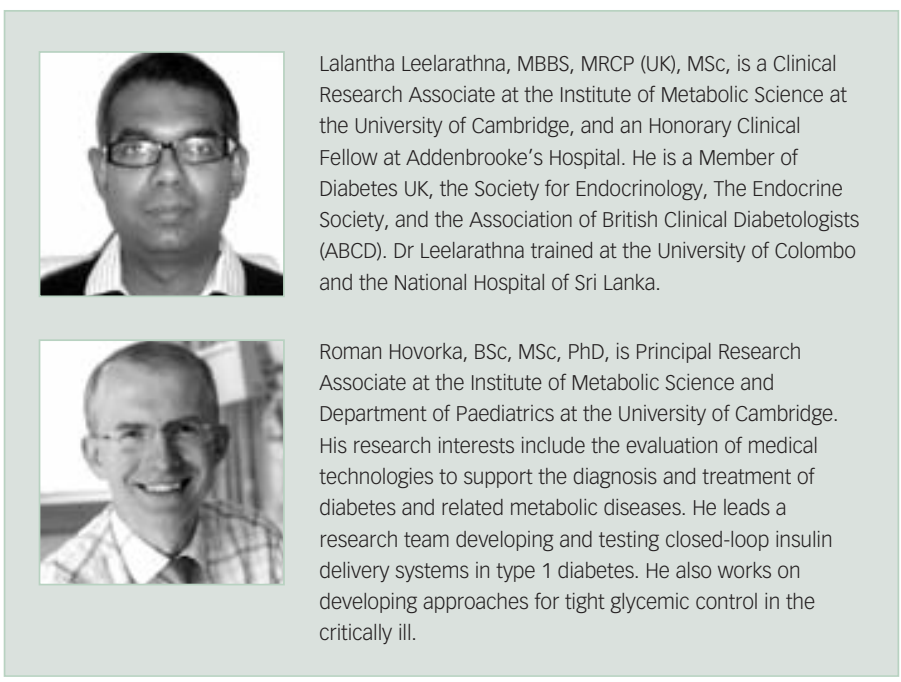

1. International Diabetes Federation, The Diabetes Atlas, Fourth Edition, Brussels, 2009.

2. The DIAMOND Project Group, Diabet Med, 2006;23: 857-66.

3. Patterson CC, et al., Lancet, 2009;373:2027-33

4. Forouhi NG, et al., Diabet Med, 2006;23:189-97.

5. Daneman D, Lancet, 2006; 367:847-58.

6. Shamoon H, et al., N Eng/ J Med, 1993;329:977-86.

7. Stettler C, et al., Am Heart J, 2006;152:27-38.

8. Cryer P, Diabetologia, 2002:45:937-48.

9. Juvenile Diabetes Research Foundation Continuous Glucose Monitoring Study Group, Diabetes Care, 2010;33: 1004-8.

10. Buckingham B, et al., J Pediatr, 2007;151:388-93.

11. Bode B, et al., Diabetes Care, 2005;28:2361-6.

12. McGarraugh G, Diabetes Technol Ther, 2009;11(Suppl. 1): S17-S24.

13. Tamborlane WV, et al., N Engl J Med, 2008;359:1464-76

14. Kowalski A, Diabetes Technol Ther, 2009;11(Suppl. 1): S113-9.

15. Juvenile Diabetes Research Foundation Continuous Glucose Monitoring Study Group, Diabetes Care, 2009;32: 1378-83.

16. Beck RW, et al., Diabetes Care, 2009;32:2047-9.

17. Bergenstal R, et al., N Engl J Med, 2010;363:311-20.

18. Hovorka R, Diabet Med, 2006;23:1-12.

19. Steil G, Rebrin K, Expert Opin Drug Deliv, 2005;2:353-62.

20. Bailey T, et al., Diabetes Technol Ther, 2009:11:749-55.

21. Kamath A, et al., Diabetes Technol Ther, 2009;11:689-95.
22. Weinstein R, et al., Diabetes Care, 2007:30:1125-30.

23. Garg S, et al., Diabetes Res Clin Pract, 2010;87:348-53.

24. Wei C, et al., Diabet Med, 2010;27:117-22.

25. Keenan D, et al., J Diabetes Sci Technol, 2009;3:1207-14.

26. Klonoff D, Diabetes Care, 2005;28:1231-9.

27. Garg S, et al., Diabetes Technol Ther, 2009;11:65-72.

28. Kovatchev B, et al., Diabetes Care, 2008;31:1160-4.

29. McGarraugh G, Bergenstal R, Diabetes Technol Ther, 2009;11: 145-50.

30. Wilinska M, et al., J Diabetes Sci Technol, 2009:3:1109-20.

31. Bequette B, Diabetes Technol Ther, 2005;7:28-47.

32. Hovorka R, et al., Physiol Meas, 2004;25:905-20.

33. Magni L, et al., J Diabetes Sci Technol, 2007;1:804-12.

34. Parker R, et al., IEEE Trans Biomed Eng, 1999;46:148-57.

35. Albisser A, et al., Diabetes, 1974:23:389-96.

36. Clemens A, Med Prog Technol, 1979;6:91-8.

37. Marchetti G, et al., IEEE Trans Biomed Eng, 2008;55: 857-65.

38. Percival M, et al , J Diabetes Sci Technol, 2008:2:636-44. 39. Wang Y, et al., IEEE Trans Biomed Eng, 2010;57:211-9.

40. El-Khatib F, et al., J Diabetes Sci Technol, 2007;1:181-92.

41. Lee $\mathrm{H}$, et al., J Diabetes Sci Technol, 2009;3:1082-90

42. Hovorka R, et al., Lancet, 2010;375:743-51.

43. Dassau E, et al., Diabetes Care, 2010;33:1249-54.

44. Buckingham B, et al., Diabetes Care, 2010;33:1013-7.

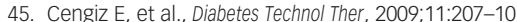

46. Elleri D, et al., Diabet Med, 2010;27:480-4.

47. Jones T, et al., N Engl J Med, 1998;338:1657-62

48. Davis E, et al., Diabetes Care, 1997;20:22-5.
49. Koltin D, Daneman D, Pediatr Diabetes, 2008:9:504-7.

50. Parekh B, Curr Diabetes Rev, 2009;5:210-5.

51. Kumareswaran K, et al., Diabet Med, 2010;27(Suppl. 1):32

52. Kumareswaran K, et al., Diabetes, 2010;59(Suppl. 1):A95.

53. Murphy HR, et al., Diabet Med, 2010;27(Suppl 1):32.

54. Steil G, et al., Diabetes, 2006;55:3344-50.

55. Weinzimer SA, et al., Diabetes Care, 2008;31:934-9.

56. Bruttomesso D, et al., J Diabetes Sci Technol, 2009;3: 1014-21.

57. Clarke W, et al. J Diabetes Sci Technol, 2009:3:1031-8.

58. Atlas E, et al., Diabetes Care, 2010;33:1072-6.

59. El-Khatib F, et al., Sci Transl Med, 2010;2:27ra27.

60. Castle JR, et al., Diabetes Care, 2010;33:1282-7.

61. Renard E, et al., Diabetes Care, 2010;33:121-7.

62. Renard E, Schaepelynck-Belicar P, Diabetes Metab, 2007;33(2):158-66

63. Chassin L, et al., Artif Intell Med, 2004:32:171-81.

64. Wilinska M, et al., J Diabetes Sci Technol, 2010;4:132-44.

65. Kovatchev B, et al. J Diabetes Sci Technol, 2009:3:44-55

66. Zisser H, et al., J Diabetes Sci Technol, 2010;4:98-103.

67. Chan A, et al., J Diabetes Sci Technol, 2008;2:844-52.

68. Man C, et al., J Diabetes Sci Technol, 2009;3:56-67.

69. Ellingsen C, et al., J Diabetes Sci Technol, 2009;3:536-44

70. Muchmore D, Vaughn D, J Diabetes Sci Technol, 2010;4: 419-28.

71. Vaughn D, et al., Diabetes Technol Ther, 2009:11:345-52.

72. Steiner $S$, et al., Diabetologia, 2008;51:1602-6.

73. Raz I, et al., Clin Ther, 2009:31:980-87.

74. Heinemann L, Diabetes Technol Ther, 2002;4:673-82. 